\title{
ARTICLE OPEN \\ Optical signatures of energy gap in correlated Dirac fermions
}

\author{
Ece Uykur ${ }^{1,2}$, Weiwu $\mathrm{Li}^{1}$, Christine A. Kuntscher ${ }^{2}$ and Martin Dressel $\mathbb{i D}^{1}$
}

Dirac fermions attract considerable interest for several years and tremendous efforts are devoted to unveil the Dirac/Weyl semimetallic state in real crystalline systems. The behavior of Dirac fermions under strong correlations and in the proximity of other ordered states is under particular scrutiny as robust experimental signatures are scarce. $a$-(BEDT-TTF) ${ }_{2} l_{3}$ constitutes a superior model in this regard because the Dirac state occurs next to an electronically ordered ground state enabling us to investigate and deliberately vary the exotic properties in correlated Dirac fermions. The charge-ordered insulator gradually evolves to a metal when pressure is applied, and at low temperatures the electronic bands form tilted Dirac-like cones. Here, we present systematic lowtemperature infrared experiments on $a$-(BEDT-TTF) ${ }_{2} l_{3}$ in an extended pressure range. A metallic state with a frequency-independent optical conductivity indicates the coexistence of the trivial and massless Dirac electrons. We discover the opening of an energy gap due to correlated Dirac fermions at the boundary to the insulating state; it is gradually suppressed when pressure increases. The unique possibility of tuning the correlated Dirac state provides unprecedented insight into this novel electronic state and yields information relevant for Dirac electron systems in general.

npj Quantum Materials (2019)4:19; https://doi.org/10.1038/s41535-019-0158-z

\section{INTRODUCTION}

Understanding the emergence and behavior of Dirac electrons in real crystalline systems is one of the central topics in current solid state research. Starting with graphene, many two- and threedimensional materials with Dirac-like linear dispersion have been discovered and investigated by employing various experimental probes. Based on these efforts, a better picture of this unique state was attained; and now more complicated situations come under scrutiny, such as correlations among the Dirac electrons and the interaction with other ground states.

Recently, the molecular conductor $a$-(BEDT-TTF) $)_{2} l_{3}$ attracted considerable attention from the experimental and theoretical side because of the particular properties of it electronic bands. Among the class of quasi-two-dimensional organic conductors, $a$-(BEDTTTF) ${ }_{2} l_{3}$ is probably the most studied compound thanks to its rich phase diagram. At ambient conditions a pronounced and sharp metal-insulator transition is observed at $T_{\mathrm{CO}}=135 \mathrm{~K}^{1}$ that eventually was explained by charge order; ${ }^{2}$ by now the ambient-pressure state of electronic ferroelectricity is rather well understood. ${ }^{3}$ Applying external pressure the insulator can be tuned to a zero-gap electronic state, and even superconductivity is reached. ${ }^{4} a$-(BEDT-TTF) ${ }_{2} l_{3}$ is the first realization of a two-dimensional multilayer massless Dirac fermion bulk system. ${ }^{5}$ The Dirac-fermion state has been proposed via different theoretical approaches ${ }^{5-9}$ and hints of the Dirac state were given by various experimental methods, such as dc transport, ${ }^{10,11}$ interlayer magnetoresistance, ${ }^{12,13}$ quantum Hall effect, ${ }^{14}$ optical, ${ }^{15,16}$ and nuclear magnetic resonance (NMR) spectroscopy. ${ }^{17,18}$ The exotic behavior of the Dirac electrons is distinct from two-dimensional graphene in many aspects and a complete understanding is still lacking.

Mott-Hubbard physics governs the ambient pressure state and enables us to gradually tune the charge order via external pressure in this system; this way we can explore correlations emerging at the Dirac points. Dirac-electron systems are subject to strong electronic correlations due to the unscreened long-range Coulomb interaction arising with the vanishing density of state around the Dirac point. ${ }^{19}$ The correlation strength can be controlled by the Coulomb coupling constant $a \approx e^{2} / \epsilon \hbar v_{F}$, which is the ratio of the Coulomb potential and the electron kinetic energy ( $\epsilon$ is the dielectric constant and $v_{\mathrm{F}}$ is the Fermi velocity). It was predicted that when $a$ is above some critical value, the massless Dirac electrons become massive with an energy gap opening near the Dirac point; ${ }^{20}$ a gap size of $100 \mathrm{meV}$ was indeed observed, for instance, in graphene. ${ }^{21}$ In the case of $a$-(BEDTTTF) ${ }_{2} l_{3}, v_{F}$ is one order of magnitude smaller compared to graphene. ${ }^{22}$ Therefore the interactions are expected to be much significant, which have been experimentally shown as an anomalous upturn in dc resistance $\rho(T)^{11}$ and spin susceptibility, ${ }^{18}$ and they strongly modified $v_{\mathrm{F} .}{ }^{17}$

Infrared spectroscopy is the superior method for studying gaps in the electronic structure and learning about the responsible interactions. The appearance of Dirac nodes in the optical spectra has been discussed for two- and three-dimensional systems theoretically and shown experimentally on several examples. ${ }^{23,24}$ The situation is expected to be more complex in $a$-(BEDT-TTF $)_{2} l_{3}$ because the Dirac cones are tilted; ${ }_{i}^{4}$ nevertheless optical investigations should give valuable insight into the nature of the Dirac state and the role of the electron-electron interactions. By our study, several contributions to the electronic interactions are resolved in a systematic way. Hence, we unambiguously confirm the existence of the Dirac state and provide the missing information on the relevant energy range and pressure evolution of the proposed correlations.

\footnotetext{
${ }^{1}$ Physikalisches Institut, Universität Stuttgart, 70569 Stuttgart, Germany and ${ }^{2}$ Experimentalphysik 2, Universität Augsburg, 86159 Augsburg, Germany Correspondence: Ece Uykur (ece.uykur@pi1.physik.uni-stuttgart.de)

These authors contributed equally: Ece Uykur, Weiwu Li
}

Received: 4 September 2018 Accepted: 25 March 2019

Published online: 30 April 2019 


\section{RESULTS AND DISCUSSION}

Samples

The organic conductor $a$-(BEDT-TTF $)_{2} l_{3}$ consists of alternatively conducting layers of BEDT-TTF (bis-(ethylenedithio)tetrathiafulvalene) molecules and insulating layers of tri-iodine anions $\left(I_{3}^{-}\right)$, as shown in Fig. 1. Since the out-of-plane conductivity (c-axis) is 1000 times smaller than the one along the in-plane ( $a, b$-axes), this compound can be well considered as quasi two dimensional system, where the bulk electronical state can be described mostly as the contribution of the electron hoping in the $a b$-plane due to the BEDT-TTF molecular orbital overlap.

In Fig. 2a, we demonstrated the overall fit of our $0.3 \mathrm{GPa}$ spectrum at room temperature. The spectrum at $p=0.3 \mathrm{GPa}$ consists of a strong mid-infrared absorption band around $3000 \mathrm{~cm}^{-1}$ and an overdamped Drude contribution. Owing to the complex structure of this material in addition to the electronic excitations, many small vibration modes are expected ${ }^{25,26}$ between 400 and $1300 \mathrm{~cm}^{-1}$, originating from the electron-molecular vibrational-coupled $\mathrm{A}_{g}$
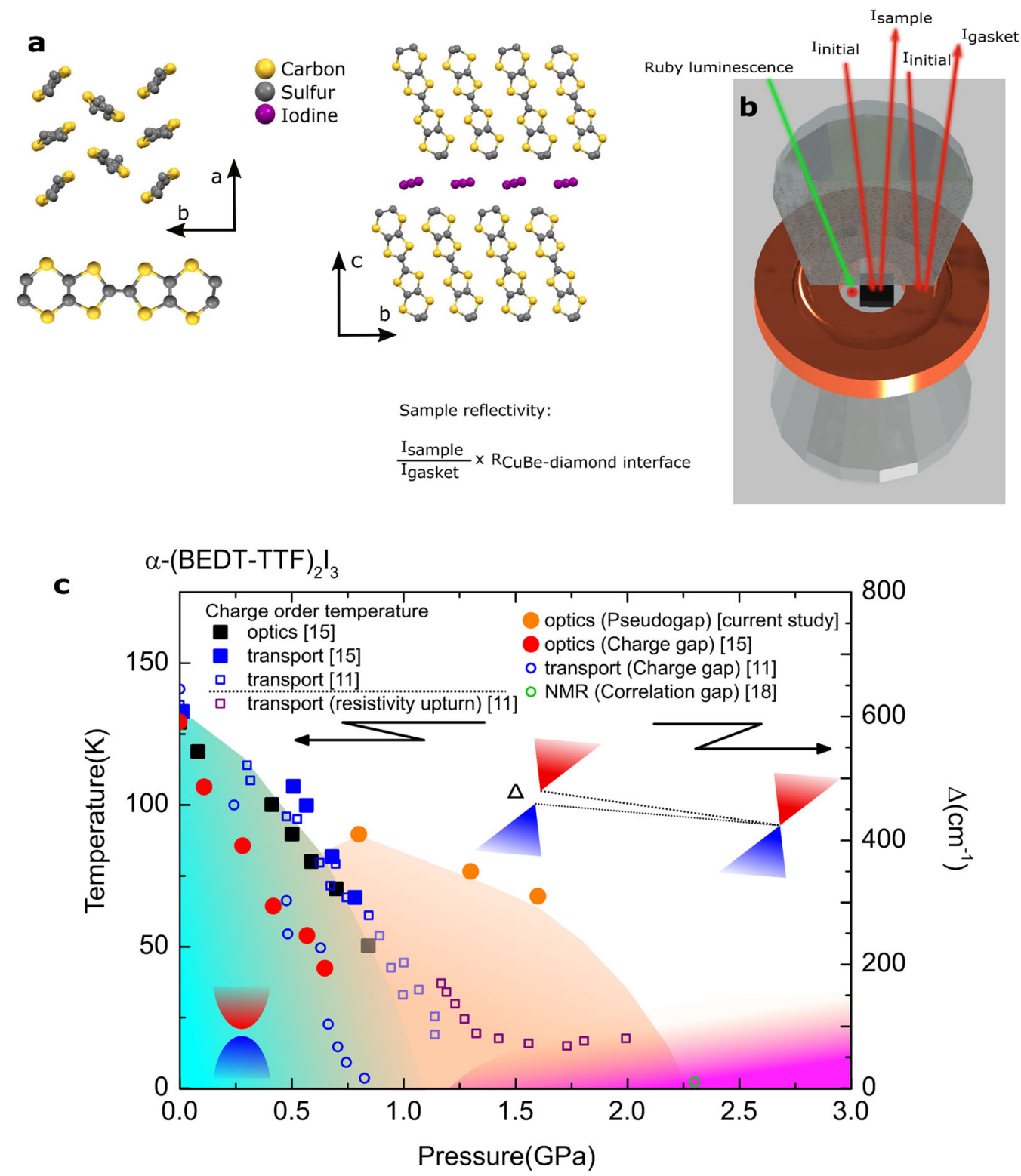

Fig. $1 a$-(BEDT-TTF $)_{2} l_{3}$ crystals, phase diagram, and infrared spectroscopy in a diamond anvil cell. a Crystal structure of $a$-(BEDT-TTF) ${ }_{2} l_{3}$. b Measurement of the reflectivity in a diamond anvil cell (DAC). CuBe gasket have been used for a reference and the obtained reflectivity have been corrected for the reflectivity of the gasket. Ruby luminescence have been used as a pressure determination technique insitu at each temperature. c Phase diagram of $a$-(BEDT-TTF) ${ }_{2} l_{3}$. Left scale shows the charge order transition temperature obtained from various methods (squares) and right scale represents charge gaps obtained via thermal activation and optics (circles). In this case they are not related with mean field theory; therefore, we scale the $p=0$ data points to combine both. The Dirac state (correlated and massless Dirac fermions) related scaling also added to the phase diagram. There is a pressure range (between $\sim 0.8$ and $\sim 1.3 \mathrm{GPa}$, shown lighter), where the accuracy of the optical measurements are decreased (due to the low-frequency limit) and the low-temperature upturn of the resistivity due to metal insulator transition and of different origin cannot be distinguished clearly. This low-temperature upturn has been identified as the Dirac state and extends to high-pressure regime, ${ }^{15}$ as also evident from our optical measurements (up to $4 \mathrm{GPa}$ ), while the charge order-Dirac state boundry can be identified with the correlated Dirac electrons. At $\sim 2.3 \mathrm{GPa}$ a very small-energy scale of these correlations has been identified via NMR experiments, ${ }^{18}$ while the current optical study also put forward the pressure dependence of the correlated Dirac state 


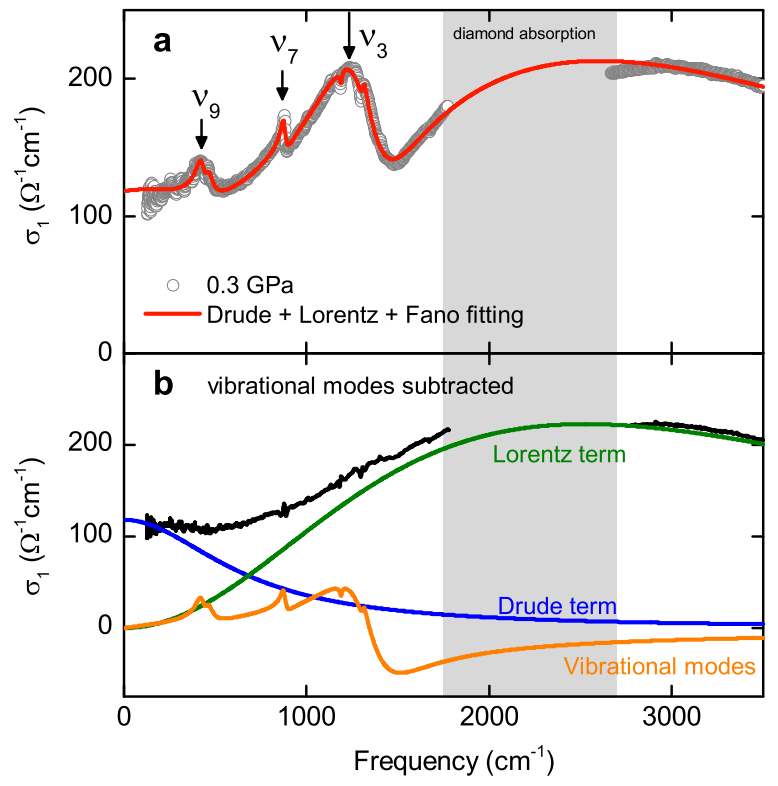

Fig. 2 Optical conductivity fitting. a Experimental optical conductivity and the Drude (low-energy incoherent carrier contribution) + Lorentz (high-energy excitations) + Fano modes (vibrational features) fitting at $0.3 \mathrm{GPa}$ as example. Three main vibration modes $\left(v_{3}\right.$, $v_{7}$, and $v_{9}$ ) are labeled consistently with the literature. b Optical conductivity spectra after the vibrational features are subtracted is given with the different contributions. Gray shaded area demonstrate the spectral region, where the diamond absorption due to the pressure cell is dominated

modes. ${ }^{27}$ We can consistently resolve the ones with strong enough contributions. Especially three of these vibration modes are quite large and contribute to the spectra significantly, namely, $v_{3}, v_{7}$, and $v_{9}$ modes. The energy range of the determined vibration modes are in accord with literature data.

Since these modes obscure the electronic background, we fitted our conductivity spectra by a Drude component for the lowenergy free carrier contribution, a Lorentz component for the high-energy mid-infrared absorption, and Fano terms for the vibration modes; an example is given in Fig. 2a and the individual contributions, Drude (blue), high-energy Lorentz (green), and vibration modes (orange), are given in Fig. $2 b$. For the further analysis vibration modes have been subtracted as also plotted in Fig. 2b. The Fano-shaped vibration modes have been well established in this molecular compound, as well as other counterparts. ${ }^{26}$ Therefore, such fitting procedure is justified.

Room-temperature optical conductivity

Figure 3 a displays the room-temperature optical conductivity of $a$ (BEDT-TTF $)_{2} l_{3}$ for various pressures. The overall shape and the absolute value of $\sigma_{1}(\omega)$ measured at lowest pressure is very similar to previous results recorded under ambient conditions, ${ }^{15,28}$ indicating the high-quality and reproducibility of our experiments.

Below $1000 \mathrm{~cm}^{-1}, \sigma_{1}(\omega)$ becomes enhanced by pressure with a progressive change from a broad to a narrower Drude behavior above $0.8 \mathrm{GPa}$, suggesting a bad metal- to good-metal transition, i.e., from incoherent to more coherent transport. The mid-infrared band persists up to values as high as $4.0 \mathrm{GPa}$ with a gradual suppression in intensity. The enhancement of the low-frequency $\sigma_{1}(\omega)$ is in line with the pressure-dependent dc-resistivity measurements, ${ }^{11,15}$ suggesting that even at room temperature the electronic state changes with applied pressure.

To characterize the charge dynamics under pressure quantitatively, the spectral weight (SW) of the zero-frequency and mid-
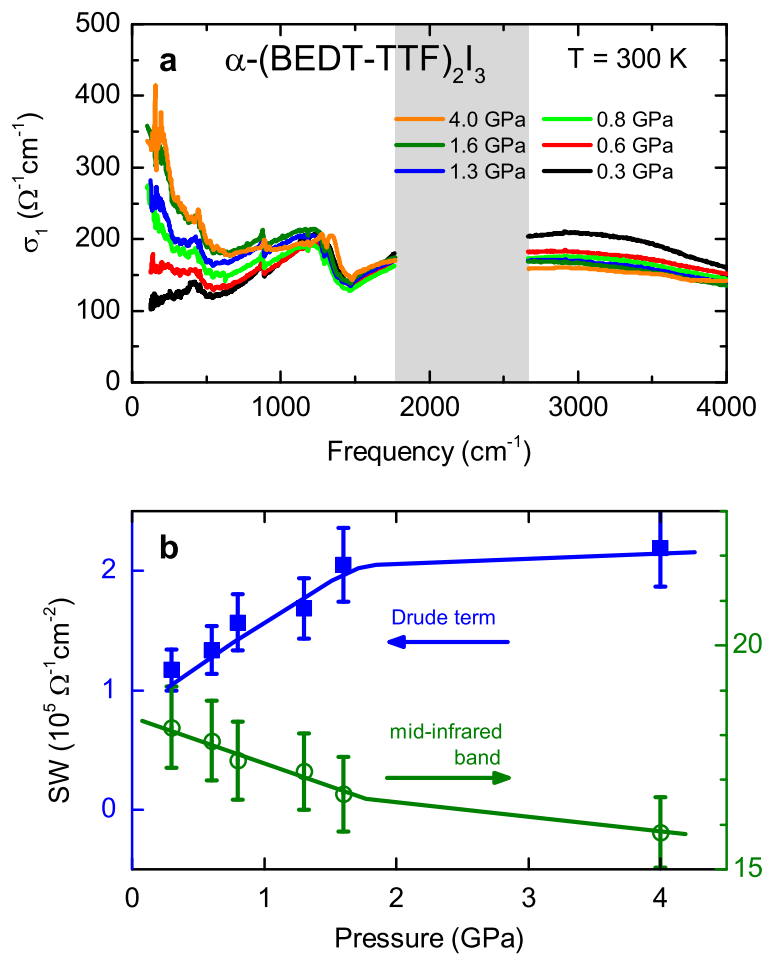

Fig. 3 Pressure-dependent room temperature optical conductivity. a Pressure evolution of the room-temperature optical conductivity of $a$-(BEDT-TTF $)_{2} I_{3}$. b Pressure dependence of the spectral weight (SW) for Drude and Lorentz components at $T=300 \mathrm{~K}$; the blue squares (Drude) refers to the left axis, the open green dots (Lorentz) correspond to the right axis

infrared bands is plotted in Fig. 3b. For the spectral weight analysis we used the contributions obtained from the Drude-Lorentz fits. With increasing pressure a systematic transfer of spectral weight occurs from high energies to the Drude component; this trend seems to saturate above $p \approx 2 \mathrm{GPa}$. The energy range for which the spectral weight transfer is observed is comparable to the onsite Coulomb repulsion $U \approx 0.4 \mathrm{eV}^{7}$ this is reminiscent of the behavior observed in the bandwidth-controlled metal-insulator in Mott insulators ${ }^{29}$ and also occurs in other charge-ordered organic compounds. ${ }^{27,30}$ The mid-infrared conductivity arising from transitions between the bands split by electronic correlations; the enhancement of the Drude-like response corresponds to the overlap of the two bands that increases with pressure. ${ }^{31}$ Hence, our room-temperature optical studies provide strong evidence that electronic correlations are important for $a$-(BEDT-TTF) ${ }_{2} l_{3}$. This conclusion is supported by recent dc transport ${ }^{11}$ and NMR measurements; ${ }^{17,18}$ our findings are also consistent with conclusions based on the extended Hubbard model, which considers onsite and inter-site Coulomb repulsion. ${ }^{7,9,32}$

\section{$T$ - and $p$-dependent optical conductivity}

The temperature-dependent conductivity spectra at various pressures are displayed in Fig. 4. Previously we showed ${ }^{15}$ that the low-pressure regime $(0-0.8 \mathrm{GPa}$ at $8 \mathrm{~K})$ is characterized as a charge-ordered ground state at low temperatures: The non-zero but relatively small spectral weight below $400 \mathrm{~cm}^{-1}$ at $T=150 \mathrm{~K}$ is suppressed when the metal-insulator transition is reached at $T_{\text {CO }}$ with a clear gap opening, marked by the green arrow in Fig. 4 e. On the other hand, the temperature evolutions of $\sigma_{1}(\omega)$ at pressures above $0.8 \mathrm{GPa}$ are qualitatively different. When one compare the room temperature and the $90 \mathrm{~K}$ spectra, $a$-(BEDTTTF) ${ }_{2} l_{3}$ exhibits metallic behavior with a characteristic spectral 

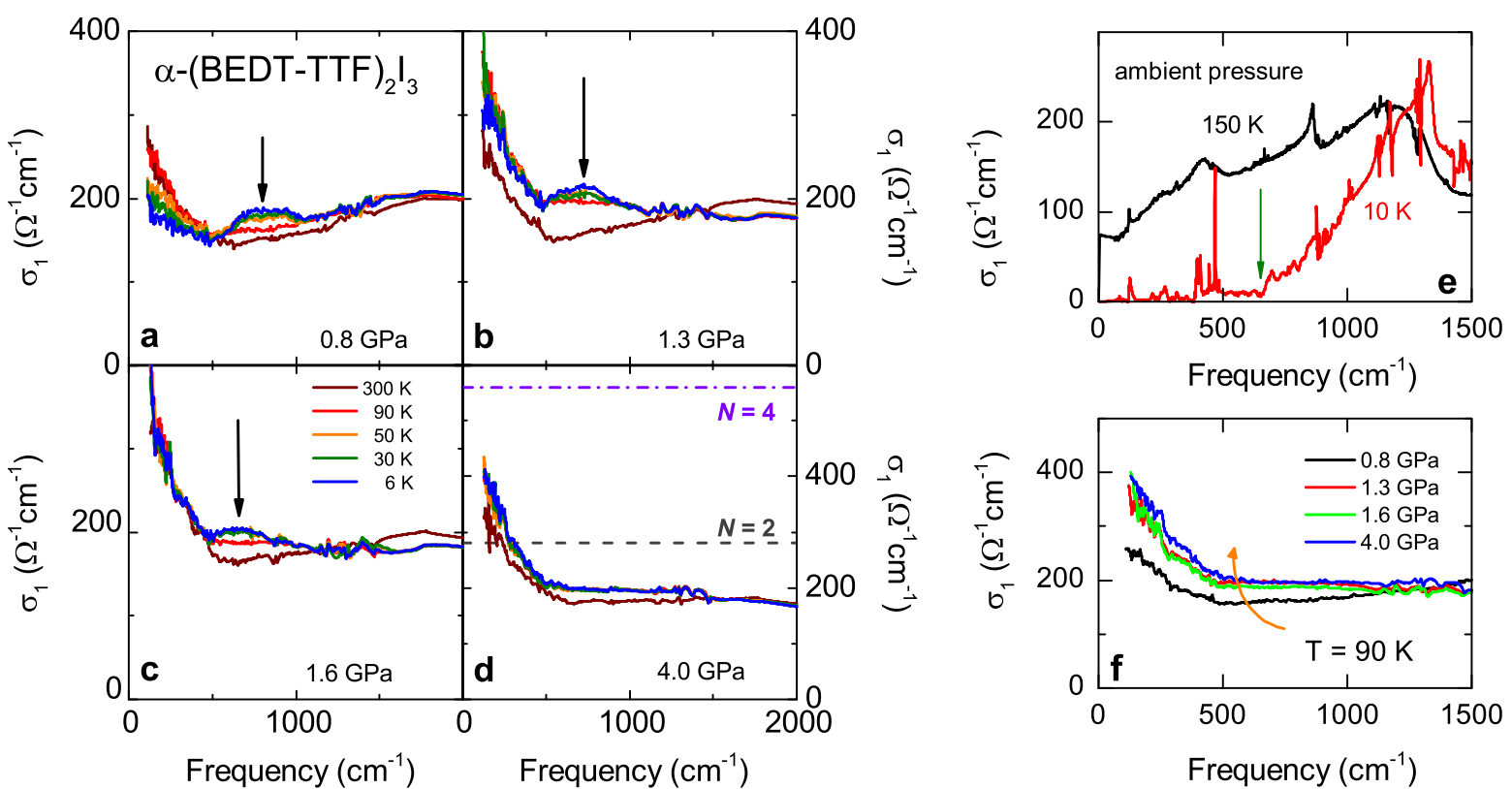

Fig. 4 Temperature- and pressure-dependent optical conductivity. a-d Temperature-dependent conductivity at $p=0.8,1.3,1.6$, and 4.0 GPa. The black arrows mark the absorption feature due to excitations across an energy gap. In $\mathbf{d}$ the bulk conductance corresponding to $G(\omega) / c$ for $N=2$ and 4 is indicated by dashed gray and dash-dotted purple lines, respectively, where $c \approx 1.7 \mathrm{~nm}$ is the $c$-axis lattice constant. ${ }^{1,39}$ e The ambient-pressure $\sigma_{1}(\omega)$ above and below the metal-insulator transition $T_{\text {co }}$ clearly shows the opening of the charge-order gap (green arrow). f $\sigma_{1}(\omega, T=90 \mathrm{~K})$ for various pressures; between $p=0.8$ and $1.3 \mathrm{GPa} \sigma_{1}(\omega)$ becomes frequency independent above $500 \mathrm{~cm}^{-1}$

weight transfer towards low energies as $T$ decreases, and a Drude-like response below $500 \mathrm{~cm}^{-1}$ (Fig. $4 \mathrm{~b}-\mathrm{d}$ ). The energy range of this transfer $(\sim 0.5 \mathrm{eV}$, see Fig. $\mathrm{S} 3$ for the full transfer range) suggests strong modifications of the trivial bands (nonDirac bands, possibly possess parabolic dispersion) upon pressure. This metallic behavior persists up to the maximum pressure measured $(4.0 \mathrm{GPa})$, indicating that the ground state above $p \approx 0.8 \mathrm{GPa}$ is distinct from the charge-ordered state; with the existence of the $\omega$-independent optical conductivity (Fig. 4f) this state can be identified where Dirac electrons exist.

Temperatures below $90 \mathrm{~K}$ reveal a density wave-like behavior, namely the suppression of the low-energy optical conductivity and a spectral weight transfer to the higher frequency range developing an absorption like feature at around $800 \mathrm{~cm}^{-1}$ that we assign to excitations across an energy gap and defined as a "pseudogap" opening. This behavior can be most clearly seen at $0.8 \mathrm{GPa}$ spectra, however, with increasing pressure, this structure shifts to lower energies and becomes gradually suppressed. We can unambiguously trace the gap feature up to $p=1.6 \mathrm{GPa}$; but at $4.0 \mathrm{GPa}$ we cannot resolve the band anymore within the accessible temperature and energy range.

One should closely inspect the $\sigma_{1}(\omega, T)$ at $p=0.8 \mathrm{GPa}$ (Fig. 4a), while this pressure specifically sits at the boundary between the insulator and Dirac metal state (See Supplementary Section 1 for additional information). The existence of the Drude-like behavior clearly distinguish this state than the insulating state where one can see a charge gap. The density-wave like behavior is common with higher pressure spectra put forward the similarities, however, lack of $\omega$-independent optical conductivity indicate that $0.8 \mathrm{GPa}$ range is not completely belong to the highpressure Dirac state either. Hence, we identify this pressure as somewhat boundary pressure between charge ordered ground state and the high-pressure Dirac state.

Based on our optical study, the previously observed anomalous upturn in the pressure-dependent transport ${ }^{11}$ is now be identified as the gap feature in the metallic ground state. Here we can further discuss about the energy range of the observed pseudogap feature.
A similar behavior is reported for density-wave systems with an only partially-gapped Fermi surface, ${ }^{33,34}$ or Mott insulators with metallic carriers remaining; ${ }^{35,36}$ hence we have to discuss the origin of the peak structure and in-gap absorption in more detail.

Optical gap formation due to correlated Dirac electrons

Two-dimensional massless Dirac electrons manifest themselves in a frequency-independent conductivity; the interband conductivity per layer should be a universal constant $G(\omega)=N \pi G_{0} / 4$, with $N$ the number of non-degenerate Dirac cones and $G_{0}=2 e^{2} / \hbar$ is the quantum conductance. Such peculiar $\sigma_{1}(\omega)$ behavior has been discussed theoretically and experimentally, for instance, in monolayer graphene ${ }^{23}$ and quasi-two-dimensional graphite. ${ }^{24}$ As displayed in Fig. 4f, $\sigma_{1}(\omega)$ becomes constant between 400 and $1200 \mathrm{~cm}^{-1}$ as pressure rises from 0.8 to $1.3 \mathrm{GPa}$ and then remains unchanged. The flat conductivity observed in this large spectral region is taken as strong evidence for massless Dirac fermions; the observed value is quite close to the predicted conductance for $N=2$ (Fig. 4d). In comparison to the case of graphite and graphene, the energy region of the $\omega$-independent conductivity is limited due to the band structure, in agreement with theory ${ }^{32}$ and NMR experiments. ${ }^{17,18}$

With the modifications of the trivial bands and the existence of the Dirac state it is rather challenging to analyze the low-energy behavior of $\sigma_{1}(\omega)$. Since the Drude component below $300 \mathrm{~cm}^{-1}$ does not show a significant $T$-dependence above $1.3 \mathrm{GPa}$, there should be some counter interacting mechanisms. Since the trivial bands in this material cannot be ignored, one would expect to see the increase of the Drude intensity with decreasing temperatures given for a regular metallic system. On the other hand, we propose two contributions that can cause the decrease of the intraband transitions in the context of Dirac systems: (i) the correlated Dirac electrons and (ii) the thermally excited massless Dirac electrons. The former contribution should become stronger as the temperature decreases. For a pure Dirac system with the chemical potential located exactly at the Dirac point, the spectral weight of the zeroenergy response should decrease upon cooling. ${ }^{24}$ Magnetotransport 
and optical measurements ${ }^{15,16,22}$ indicate the coexistence of correlated and massless Dirac carriers. This coexistence can also explain the deviations from the quantum resistance observed in $\rho(T)$ between 300 and $10 \mathrm{~K}^{10,11,15}$ The tilting of the Dirac cones present in $a$-(BEDT-TTF $)_{2} l_{3}{ }^{17,18}$ leads to a strong anisotropy of the Fermi velocity and modifies the interband transitions of the Dirac electrons. In accord with Suzumura et al. ${ }^{37}$ we expect an effect only in the $\mathrm{THz}$ optical response (around $20 \mathrm{~cm}^{-1}$ ), and not at higher energies where we observe $\sigma_{1}(\omega)=$ const.

Our optical results not only provide compelling evidence of interacting Dirac fermions, but give insight into the actual density of electronic states. For a better illustration, in Fig. 5 we plot the difference of the conductivity between $T=6$ and $90 \mathrm{~K}$. The opening of a pseudogap is well identified as peaks in $\Delta \sigma_{1}(\omega)$ and indicated by arrows. From a proposed density of states as displayed in the inset for the different pressure values, a fit to the difference of the optical conductivity (solid lines) can be obtained. In our model we assume the optical joint density of states to be proportional to $\omega^{2} \epsilon_{2}=4 \pi \omega \sigma_{1}(\omega)$ and neglect the effect of the matrix element. ${ }^{38}$ At low temperatures $(T=6 \mathrm{~K})$ a pronounced pseudogap is present with a significant reduction of the density of states. The spectral weight missing below the gap is recovered at higher energies, which manifest itself with a spectral weight transfer from low to high energies causing an absorption peak in the optical spectra. We define the energy of this peak structure as $2 \Delta$ (shown with arrows in Figs. 4 and 5), and for the pseudogap we obtain $\Delta=410,350$, and $310 \mathrm{~cm}^{-1}$ at $p=0.8,1.3$, and $1.6 \mathrm{GPa}$ respectively; and it seems to be completely suppressed at $4.0 \mathrm{GPa}$. As can be seen from the fits in Fig. 5, with rising pressure, the assumption of the decrease of $\Delta$ and suppression of the pseudogap by gradual filling in states from above can reproduce the experimental finding satisfactorily.

These findings can be compared to the gap values estimated by Khveshchenko ${ }^{20}$ using:

$$
\Delta=v_{F} \hbar \wedge \exp [(-2 \pi+4 \arctan \sqrt{2 \tilde{a}-1}) /(\sqrt{2 \tilde{a}-1})] / k_{B},
$$

where $\tilde{a}=a /(1+N \pi a / 8 \sqrt{2})$, and $\Lambda=0.667 \AA^{-1}$ is a momentum cutoff at the inverse lattice constant. ${ }^{39}$ For our estimation the dielectric constant $\epsilon_{\infty} \approx 4$ was directly extracted from the highfrequency limit (See Supplementary Section 3); the Fermi velocity $v_{\mathrm{F}} \approx 2.4-10 \times 10^{4} \mathrm{~ms}^{-1}$ was taken from refs. ${ }^{8,10,17}$. With the assumption of $N=2$, we obtain $\Delta \approx 300 \mathrm{~cm}^{-1}$, in good agreement with our optical data. There are two non-degerate Dirac cones $(N=4)$ predicted, ${ }^{6,8}$ which merge into one at high pressure $(N=$ $2)^{7}$ In combination of the observed universal constant conductance and the pseudogap, our present optical study yields strong evidence of a single Dirac cone. In a theoretical study of uniaxial pressure, ${ }^{40}$ it was recently predicted that $a$-(BEDT-TTF $)_{2} l_{3}$ exhibits three-quarter Dirac points; further experiments have to show whether this is of relevance for the optical conductivity. Our conclusion from the optical spectra are further supported by suggestions that the massless Dirac electrons are strongly correlated due to the unscreened long-range Coulomb repulsion giving rise to an anomalous increase in $\rho(T),{ }^{11}$ strong modification of the Fermi velocity ${ }^{17}$ and excitonic mass generation at low $T .^{18}$

Theoretically, a gap opening due to spin-orbit coupling and/or merging of Dirac cones under strain in 2D topological organic materials have been discussed. ${ }^{41-43}$ Especially considering the heavy mass of the iodine in the current material, one would argue that the observed pseudogap is the direct bandgap due to spinorbit coupling. However, the predicted energy gap in these conditions were on the order of 1-2 meV and is well below the energy range relevant for the pseudogap. The observed densitywave like behavior could also be explained by the partial gap opening at the Fermi surface due to trivial bands, for instance via nesting, and we cannot rule out this possibility completely. However, as summarized in Fig. 1b, the scenario of the correlated

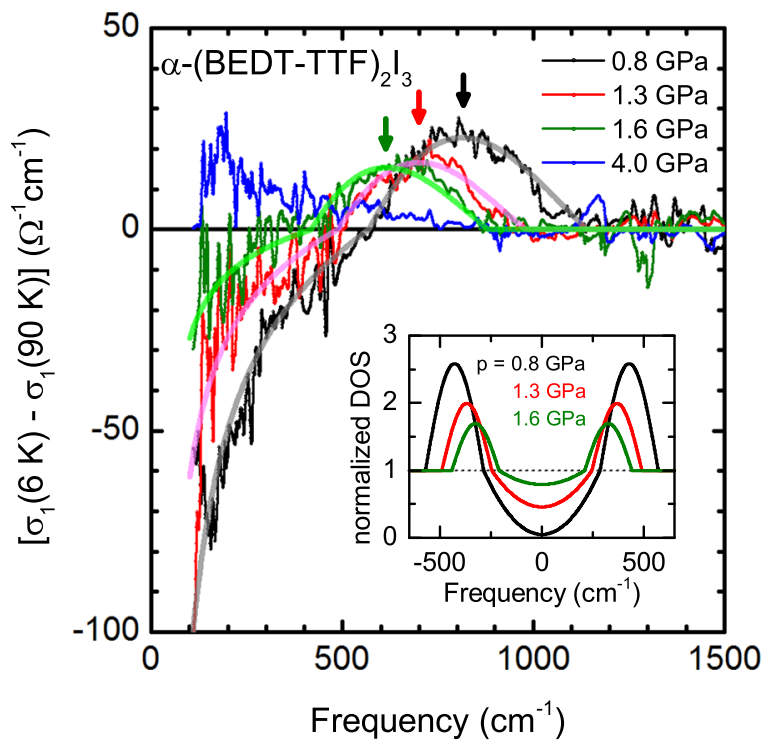

Fig. 5 Pressure-dependent difference spectra demonstrating the pseudogap behavior. Pressure dependence of the changes in the optical conductivity between $T=90$ and $6 \mathrm{~K}$. The peak structure indicated by the arrows shifts toward lower energies and diminishes as pressure increases. The solid lines correspond to $\Delta \sigma_{1}(\omega)$ calculated from the electronic density of states given in the inset. The pseudogap closes by pressure and is completely absent at $p=4 \mathrm{GPa}$

Dirac electrons fits the general picture presented with several other high-pressure studies done on the same material.

Therefore, we proposed the current explanation, and the conclusions drawn from our pressure-dependent optical investigations are summarized in the schematic density of states and $\sigma_{1}(\omega)$ sketched in Fig. 6. At room temperature and for low pressure, $a$-(BEDT-TTF) $)_{2} l_{3}$ is a bad metal with a very broad Drude contribution to $\sigma_{1}(\omega)$ originating from the thermally excited electrons at the Fermi energy. Upon cooling, these excitations freeze out and a clear charge-order gap develops in the optical conductivity. With rising pressure these bands approach each other and overlap, leading to a more-or-less narrow Drude contribution (dark green curve). As we cool down, the edges of the two bands develop linear dispersions. In the high-pressure range $\sigma_{1}(\omega)$ consists of three components: (i) a low-energy Drude response (red area), (ii) a frequency-independent conductivity due to the Dirac electrons (blue area) and (iii) a mid-infrared band arising with the incoherent transitions due to on-site and inter-site Coulomb repulsion (green area). With further cooling, electronic correlations cave in a pseudogap with states piling up at the edges. As a result, the Drude spectral weight is transferred to finite energies, leading to local peaks in $\sigma_{1}(\omega)$ around $800 \mathrm{~cm}^{-1}$ (blue curve). Note, the behavior is distinct from superconductivity, where the missing spectral weight condenses in a $\delta(\omega=0)$ peak. $^{38}$ The phase diagram as a function of external pressure displayed in the lower part of Fig. 6 can be divided into three parts: (i) the charge-order insulating state at low pressure; (ii) metallic states in the intermediate pressure regime consisting of massless Dirac electrons, next to carriers in correlation-split and trivial bands; (iii) above 4.0 GPa only the Dirac electronic state and carriers in trivial bands remain (dashed red curve). Our optical results demonstrate clear fingerprints of the electronic correlations among the Dirac electrons; the interaction can be tuned by temperature and pressure. We call for complementary spectroscopic investigations to directly confirm our findings and further efforts for a theoretical description of our observations. 


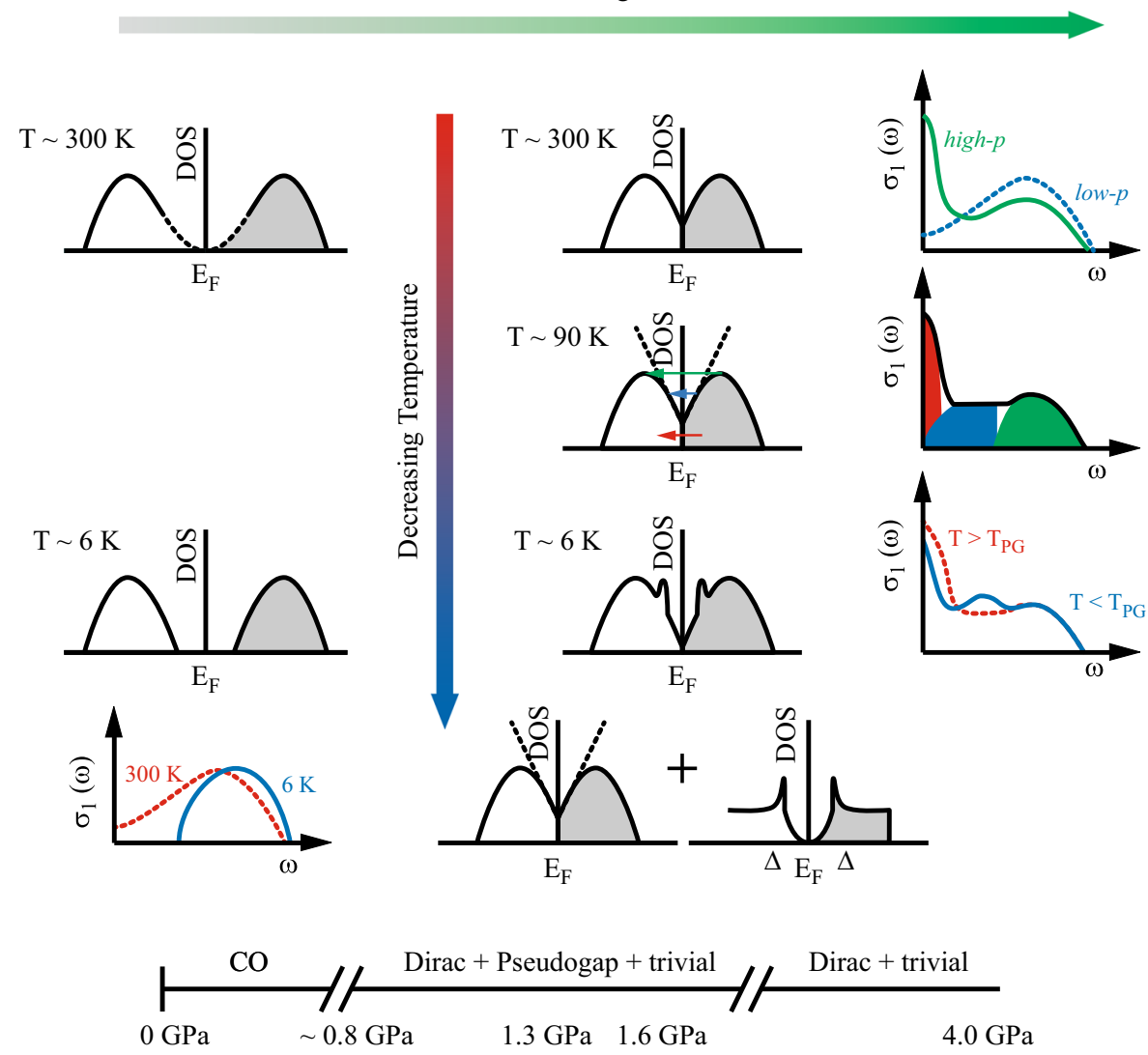

Fig. 6 The schematic diagrams for the electronic structure and corresponding optical conductivity of $a$-(BEDT-TTF $)_{2} l_{3}$. Diagrams are given for the low-pressure insulator state and the high-pressure Dirac state at various temperatures. Here, $T_{\mathrm{PG}}$ stands for the temperature, where the pseudogap starts to open. At the bottom the pressure evolution of the various electronic phases of $a$-(BEDT-TTF) ${ }_{2} l_{3}$ at low temperatures is summarized. For a detailed description refer to last paragraph

\section{METHODS}

\section{Sample preparation}

$a$-(BEDT-TTF) $)_{3}$ single crystals with the typical size of $300 \times 300 \times 60 \mu \mathrm{m}$ have been utilized for this high-pressure infrared spectroscopy study. Two pieces of flat, high-quality crystals, cut from the same big piece, were used for the far-infrared and the middle-infrared measurements. $E \| a b$-plane measurement configuration has been chosen and measurements have been conducted without a polarizer. Samples were placed inside a type-lla diamond anvil cell (DAC) ${ }^{44}$ with a culet diameter of $900 \mu \mathrm{m}$ with ruby spheres as the high-pressure manometers. Finely grounded Csl powder was employed as a quasihydrostatic pressure transmitting medium. Standart ruby luminescence technique ${ }^{45}$ has been employed to determine the pressure inside the cell at each temperature. The measured pressure range extends up to $\sim 4.0 \mathrm{GPa}$.

\section{Infrared spectroscopy}

The pressure cell is placed inside a custom made cold finger continuous flow helium cryostat (CryoVac) operating from room temperature down to $6 \mathrm{~K}$. The temperature-dependent reflectivity spectra have been measured utilizing a home-built setup ${ }^{46}$ that is coupled to a Bruker Vertex $80 \mathrm{v}$ Fourier transform infrared spectrometer. Temperature-dependent reflectivity was recorded in the frequency range from 100 to $8000 \mathrm{~cm}^{-1}$ employing standard globar light source. The reflectivity spectra were taken at the sample diamond interface, using the CuBe gasket inside the DAC as reference. Afterwards, the intensity of the measured reflectivity was normalized by the intensity reflected from the CuBe gasket at the gasketdiamond interface. The optical conductivity $\sigma_{1}(\omega)$ is obtained via KramersKronig analysis. ${ }^{47}$ Between 1700 and $2800 \mathrm{~cm}^{-1}$ the multiphonon absorption of the diamond anvil affects the measured spectra, therefore, in this energy range a linear extrapolation have been used for further analysis. Despite the bad metallicity in this system, the reflectivity shows a clear upturn. Therefore, in the low-energy range Hagen-Rubens extrapolation has been chosen. For the high-energy range a flat conductivity followed by $\omega^{-4}$ free carrier approximation have been utilized.

\section{Pseudogap feature in raw spectra}

We would like to emphasize that the low-energy suppression of the optical conductivity and a concominant high-energy peak-like structure is already visible in the as measured spectra, as well. In Fig. 7a, we plot the as measured reflectivity spectra as a function of energy and temperature for $0.8 \mathrm{GPa}$, where the vibration modes have rather dominant effect (See Supplementary Section 2 for the other pressures). With lowering temperature a clear suppression of the low-energy reflectivity with an inflection point at around $800 \mathrm{~cm}^{-1}$ (marked with an arrow) can be observed. This behavior is reflected in optical conductivity, as well (Fig. 7b) as described above. The arrow marks the peak-like structure in the presence of the vibration modes, a clear slope change below $90 \mathrm{~K}$ is well defined.

In Fig. 8a, b, we further demonstrate that this additional absorption band due to pseudogap is not an artifact of our fitting procedure and is not caused by the vibration modes. At $90 \mathrm{~K}$, we can reproduce the optical conductivity very well with the above mentioned contributions, namely, a Drude, mid-infrared Lorentz, and Fano-shaped vibration modes. With cooling down, as expected, vibration modes are getting sharper. On the other hand, the small absorption feature (marked with ${ }^{*}$ ) cannot be reproduced with taking into account only the vibration modes. We plotted the best fits in Fig. 8b w/ (red curve) and w/o (blue curve) this feature for a comparison. This mode is very broad to be an additional vibration mode, also have not been reported before.

\section{DATA AVAILABILITY}

All relevant data are available from the authors upon request. 


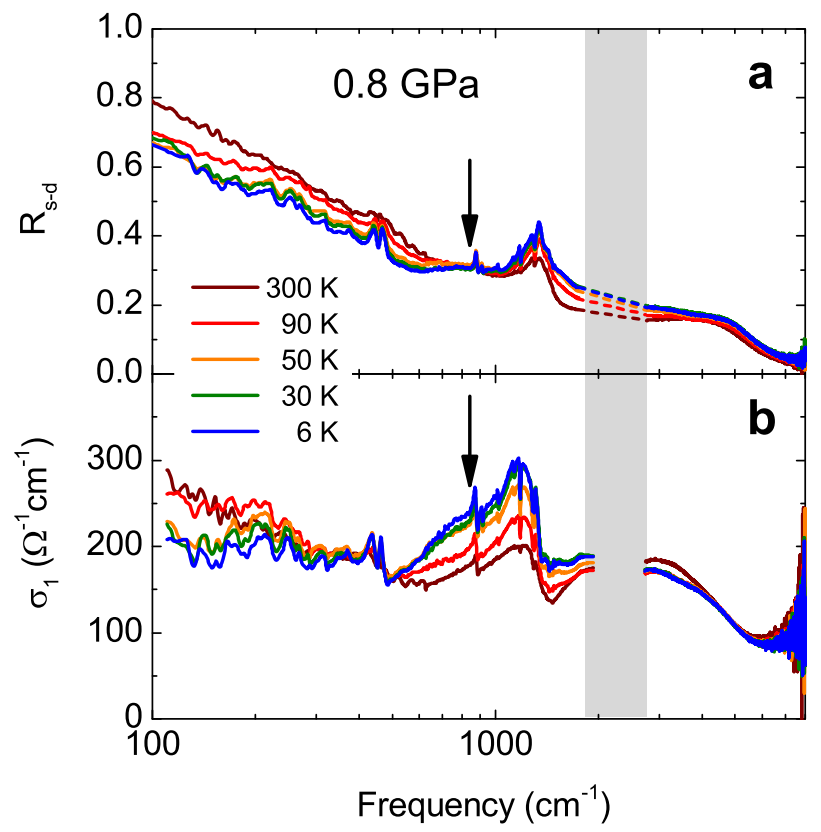

Fig. 7 Temperature-dependent reflectivity and optical conductivity at $0.8 \mathrm{GPa}$. a Reflectivity of $a$-(BEDT-TTF $)_{2} I_{3}$ at the diamond sample interface at $0.8 \mathrm{GPa}$. A systematic decrease of the reflectivity with lowering temperature is visible below the energy range marked with the arrow. b Optical conductivity spectra calculated with Kramerskronig analysis from the reflectivity spectra in a. The suppression of the low-energy optical conductivity and appearence of a peak-like structure (shown with an arrow) indicates the low energy to highenergy spectral weight transfer

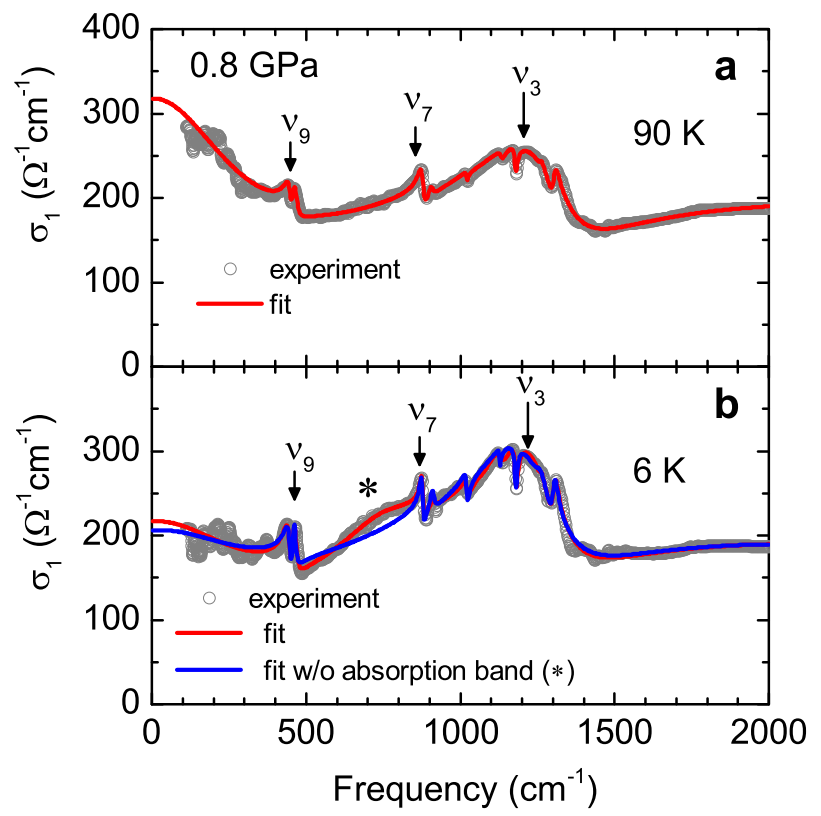

Fig. 8 Fitting procedure of the low-temperature spectra at the highpressure regime ( $0.8 \mathrm{GPa}$ as an example). a In all, $90 \mathrm{~K}$ spectra can be reproduced well with a Drude + Lorentz + Fano fitting as described in Fig. 2b. b With lowering temperature a peak-like structure starts to evolve at the frequency range $500-1000 \mathrm{~cm}^{-1}$. The best fitting attempt with only Drude + Lorentz + Fano components described previously is given as the blue curve. As one can see, the absorption feature needs to be taken into account for a good fitting (given with a red curve)

\section{ACKNOWLEDGEMENTS}

We thank A. Pronin and M. Hirata for useful discussions, D. Schweitzer for providing the single crystals, and G. Untereiner for technical support. We also acknowledge the support by the Deutsche Forschungsgemeinschaft (DFG). E.U. acknowledges the support by "Margarete von Wrangell Habilitation Programm" by the Ministry of Sciences, Research, and Arts in Baden Württemberg. E.U. and C.K. acknowledge financial support by the Federal Ministry of Education and Research (BMBF), Germany, through Grant No. 05K13WA1 (Verbundprojekt 05K2013, Teilprojekt 1, PT-DESY).

\section{AUTHOR CONTRIBUTIONS}

W.L. and E.U. contributed equally to this work and carried out the experiments and the data analysis. C.K. and M.D. supervised the work. All authors contributed to the manuscript.

\section{ADDITIONAL INFORMATION}

Supplementary Information accompanies the paper on the npj Quantum Materials website (https://doi.org/10.1038/s41535-019-0158-z).

Competing interests: The authors declare no competing interests.

Publisher's note: Springer Nature remains neutral with regard to jurisdictional claims in published maps and institutional affiliations.

\section{REFERENCES}

1. Bender, K. et al. (BEDT-TTF) ${ }_{2} l_{3}$ : A two-dimensional organic metal. Mol. Cryst. Liq. Cryst. 107, 45-53 (1984).

2. Kino, H. \& Fukuyama, H. On the phase transition of $a$-(BEDT-TTF) ${ }_{2} \mathrm{I}_{3}$. J. Phys. Soc Jpn. 64, 1877-1880 (1995).

3. Tomić, S. \& Dressel, M. Ferroelectricity in molecular solids: a review of electrodynamic properties. Rep. Progr. Phys. 78, 096501-096526 (2015).

4. Kajita, K., Nishio, Y., Tajima, N., Suzumura, Y. \& Kobayashi, A. Molecular dirac fermion systems-theoretical and experimental approaches. J. Phys. Soc. Jpn. 83, 072002-072032 (2014)

5. Katayama, S., Kobayashi, A. \& Suzumura, Y. Pressure-induced zero-gap semiconducting state in organic conductor $a$-(BEDT-TTF) ${ }_{2} I_{3}$ salt. J. Phys. Soc. Jpn. 75 054705-054710 (2006).

6. Kino, H. \& Miyazaki, T. First-principles study of electronic structure in $a$-(BEDTTTF) ${ }_{2} l_{3}$ at ambient pressure and with uniaxial strain. J. Phys. Soc. Jpn. 75, 034704-034711 (2006).

7. Kobayashi, A., Katayama, S., Suzumura, Y. \& Fukuyama, H. Massless fermions in organic conductor. J. Phys. Soc. Jpn. 76, 034711-034716 (2007).

8. Alemany, P., Pouget, J. -P. \& Canadell, E. Essential role of anions in the charge ordering transition of $a$-(BEDT-TTF) ${ }_{2} l_{3}$. Phys. Rev. B 85, 195118-195127 (2012).

9. Suzumura, Y. \& Kobayashi, A. Theory of dirac electrons in organic conductors. Crystals 2, 266-283 (2012).

10. Tajima, N. et al. Transport properties of massless dirac fermions in an organic conductor $a$-(BEDT-TTF) $)_{2} l_{3}$ under pressure. Europhys. Lett. 80, 47002-47006 (2007).

11. Liu, D. et al. Insulating nature of strongly correlated massless dirac fermions in an organic crystal. Phys. Rev. Lett. 116, 226401-226405 (2016).

12. Tajima, N. \& Kajita, K. Experimental study of organic zero-gap conductor $a$-(BEDTTTF) $)_{2} I_{3}$. Sci. Technol. Adv. Mater. 10, $024308-024314$ (2009).

13. Tajima, N. et al. Spin and valley splittings in multilayered massless dirac fermion system. Phys. Rev. B 82, 121420-121423 (2010).

14. Tajima, N., Kato, R., Sugawara, S., Nishio, Y. \& Kajita, K. Interband effects of magnetic field on hall conductivity in the multilayered massless dirac fermion system $a$-(BEDT-TTF) $)_{2} l_{3}$. Phys. Rev. B 85, 033401-033404 (2012).

15. Beyer, R. et al. Pressure-dependent optical investigations of $a$-(BEDT-TTF) $)_{2} l_{3}$ : Tuning charge order and narrow gap towards a dirac semimetal. Phys. Rev. $B$ 93, 195116-195128 (2016)

16. Peterseim, T., Ivek, T., Schweitzer, D. \& Dressel, M. Electrically induced phase transition in $a$-(BEDT-TTF) $)_{2} l_{3}$ : Indications for dirac-like hot charge carriers. Phys. Rev. B 93, 245133-245143 (2016).

17. Hirata, M. et al. Observation of an anisotropic Dirac cone reshaping and ferrimagnetic spin polarization in an organic conductor. Nat. Commun. 7, 12666-12679 (2016)

18. Hirata, M. et al. Anomalous spin correlations and excitonic instability of interacting 2d weyl fermions. Science 358, 1403-1406 (2017). 
19. Kotov, V. N., Uchoa, B., Pereira, V. M., Guinea, F. \& Castro Neto, A. H. Electronelectron interactions in graphene: Current status and perspectives. Rev. Mod. Phys. 84, 1067-1125 (2012).

20. Khveshchenko, D. V. Massive dirac fermions in single-layer graphene. J. Phys.: Condens. Matter 21, 075303-075309 (2009).

21. Zhang, $Y$. et al. Giant phonon-induced conductance in scanning tunnelling spectroscopy of gate-tunable graphene. Nat. Phys. 4, 627-630 (2008).

22. Monteverde, M. et al. Coexistence of dirac and massive carriers in $a$-(BEDT-TTF) $)_{2} l_{3}$ under hydrostatic pressure. Phys. Rev. B 87, 245110-245114 (2013).

23. Mak, K. F. et al. Measurement of the optical conductivity of graphene. Phys. Rev. Lett. 101, 196405-196408 (2008).

24. Kuzmenko, A. B., van Heumen, E., Carbone, F. \& van der Marel, D. Universal optical conductance of graphite. Phys. Rev. Lett. 100, 117401-117404 (2008).

25. Yue, $Y$. et al. Nonuniform site-charge distribution and fluctuations of charge order in the metallic state of $a$-(BEDT-TTF) ${ }_{2} l_{3}$. Phys. Rev. B 82, 075134-075141 (2010).

26. Ivek, $\mathrm{T}$. et al. Electrodynamic response of the charge ordering phase: Dielectric and optical studies of $a$-(BEDT-TTF) ${ }_{2} I_{3}$. Phys. Rev. B 83, 165128-165140 (2011).

27. Dressel, M. \& Drichko, N. Optical properties of two-dimensional organic conductors: Signatures of charge ordering and correlation effects. Chem. Rev. 104, 5689-5716 (2004).

28. Dressel, M., Grüner, G., Pouget, J. P., Breining, A. \& Schweitzer, D. Field and frequency dependent transport in the two-dimensional organic conductor $a$ (BEDT-TTF) ${ }_{2}$ l $_{3}$. J. Phys. I Fr. 4, 579-594 (1994).

29. Basov, D. N., Averitt, R. D., van der Marel, D., Dressel, M. \& Haule, K. Electrodynamics of correlated electron materials. Rev. Mod. Phys. 83, 471-541 (2011).

30. Drichko, N. et al. Electronic properties of correlated metals in the vicinity of a charge-order transition: Optical spectroscopy of $\alpha$-(BEDT-TTF $)_{2} \mathrm{Hg}(\mathrm{SCN})_{4}\left(\mathrm{M}=\mathrm{NH}_{4}\right.$, Rb,Tl). Phys. Rev. B 74, 235121-235131 (2006).

31. Rozenberg, M. et al. Optical conductivity in mott-hubbard systems. Phys. Rev. Lett. 75, 105-108 (1995).

32. Kobayashi, A., Katayama, S. \& Suzumura, Y. Theoretical study of the zero-gap organic conductor $a$-(BEDT-TTF) $)_{2} \mathrm{l}_{3}$. Sci. Technol. Adv. Mater. 10, 024309-024323 (2009).

33. Perucchi, A., Degiorgi, L. \& Thorne, R. E. Optical investigation of the chargedensity-wave phase transitions in $\mathrm{NbSe}_{3}$. Phys. Rev. B 69, 195114-195118 (2004).

34. Barišić, N. et al. Electrodynamics of electron-doped iron pnictide superconductors: Normal-state properties. Phys. Rev. B 82, 054518-054529 (2010).

35. Dumm, M. et al. Bandwidth-controlled mott transition in $\kappa$-(BEDT-TTF $)_{2} \mathrm{Cu}\left[\mathrm{NCN}_{2}\right]$ $\mathrm{Br}_{x} \mathrm{Cl}_{1-x}$ : Optical studies of correlated carriers. Phys. Rev. B 79, 195106-195116 (2009).

36. Pustogow, A. et al. newblock Quantum spin liquids unveil the genuine Mott state. Nat. Mater. 17, 773-777 (2018)
37. Suzumura, Y., Proskurin, I. \& Ogata, M. Dynamical conductivity of dirac electrons in organic conductors. J. Phys. Soc. Jpn. 83, 094705-094713 (2014).

38. Dressel, M. \& Grüner, G. Electrodynamics of Solids. (Cambridge University Press, Cambridge, 2002).

39. Kondo, R., Kagoshima, S., Tajima, N. \& Kato, R. Crystal and electronic structures of the quasi-two-dimensional organic conductor $a$-(BEDT-TTF) ${ }_{2} l_{3}$ and its selenium analogue $a$-(BEDT-TSeF $)_{2} l_{3}$ under hydrostatic pressure at room temperature. $J$. Phys. Soc. Jpn. 78, 114714-114720 (2009).

40. Kishigi, K. \& Hasegawa, Y. Three-quarter dirac points, landau levels, and magnetization in $a$-(BEDT-TTF) ${ }_{2} I_{3}$. Phys. Rev. B 96, 085430-085452 (2017).

41. Wang, Z. F., Liu, Z. \& Liu, F. Quantum anomalous hall effect in 2D organic topological insulators. Phys. Rev. Lett. 110, 196801-196805 (2013).

42. Si, C., Sun, Z. \& Liu, F. Strain engineering of graphene: a review. Nanoscale 8 , 3207-3217 (2016)

43. Winter, S. M., Riedl, K. \& Valentí, R. Importance of spin-orbit coupling in layered organic salts. Phys. Rev. B 95, 060404-060409 (2017).

44. Keller, R. \& Holzapfel, W. B. Diamond anvil device for x-ray diffraction on single crystals under pressures up to 100 kilobar. Rev. Sci. Instrum. 48, 517-523 (1977).

45. Mao, H. K., Xu, J. \& Bell, P. M. Calibration of the ruby pressure gauge to $800 \mathrm{kbar}$ under quasi-hydrostatic conditions. J. Geophys. Res. Solid Earth 91, 4673-4676 (1986).

46. Kuntscher, C. A., Huber, A. \& Hücker, M. Suppression of the charge-density-wave state in $\mathrm{Sr}_{10} \mathrm{Ca}_{4} \mathrm{Cu}_{24} \mathrm{O}_{41}$ by external pressure. Phys. Rev. B 89, 134510-134516 (2014).

47. Pashkin, A., Dressel, M. \& Kuntscher, C. A. Pressure-induced deconfinement of the charge transport in the quasi-one-dimensional mott insulator (TMTTF) ${ }_{2} \mathrm{AsF}_{6}$. Phys. Rev. B 74, 165118-165126 (2006).

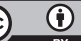

Open Access This article is licensed under a Creative Commons Attribution 4.0 International License, which permits use, sharing, adaptation, distribution and reproduction in any medium or format, as long as you give appropriate credit to the original author(s) and the source, provide a link to the Creative Commons license, and indicate if changes were made. The images or other third party material in this article are included in the article's Creative Commons license, unless indicated otherwise in a credit line to the material. If material is not included in the article's Creative Commons license and your intended use is not permitted by statutory regulation or exceeds the permitted use, you will need to obtain permission directly from the copyright holder. To view a copy of this license, visit http://creativecommons. org/licenses/by/4.0/.

(c) The Author(s) 2019 